HETEROCYCLES, Vol. , No. , , pp. -. (c) The Japan Institute of Heterocyclic Chemistry

Received, , Accepted, , Published online, . COM-06- (Please do not delete.)

\title{
SYNTHESIS OF 2,3,9,10-TETRAOXYGENATED BENZO[C]PHENANTHRIDINE DERIVATIVES VIA \\ PALLADIUM-MEDIATED ARYL-ARYL COUPLING REACTION
}

\section{Hitoshi Abe,,* Naoko Kobayashi,, ${ }^{\text {a* }}$ utaka Kadoshima, ${ }^{\text {a }}$ Yasuo Takeuchi, Takashi Harayama, ${ }^{b}$ and Yoshikazu Horino ${ }^{a}$}

a) Graduate School of Science and Engineering, University of Toyama, 930-8555, Japan, b) Graduate School of Medicine, Dentistry, and Pharmaceutical Sciences, Okayama University, 700-8530, Japan

abeh@eng.u-toyama.ac.jp

\begin{abstract}
Two 2,3,9,10-tetraoxygenated benzo[c]phenanthridine alkaloids, 1 2, originally reported as zanthoxyline and broussonpapyrine, respectively, were synthesized using the Pd-mediated intramolecular aryl-aryl coupling reaction as the key step.
\end{abstract}

\section{INTRODUCTION}

The palladium-mediated aryl-aryl coupling reaction is a powerful method to construct biaryl compounds, and many syntheses of biaryl-type natural products have been accomplished using this technique. ${ }^{1}$ Especially, intramolecular coupling is useful for the formation of polyfunctionalized heterocyclic systems. $^{2}$ Among such heterocyclic compounds, the benzo[c]phenanthridine alkaloids are recognized as an important class of natural products because of their interesting biological activities such as antitumor and antiviral activities, inhibition of DNA topoisomerase I, etc. ${ }^{3}$ In this context, we have reported several syntheses of the benzo[c]phenanthridine natural products via the intramolecular aryl-aryl coupling reaction of the benzonaphthamide derivatives. ${ }^{4}$

Recently, two unique natural benzo[c]phenanthridine alkaloids, zanthoxyline $(\mathbf{1})^{5}$ and broussonpapyrine (2), ${ }^{6}$ were independently isolated, which possess an unusual substituent pattern (Figure). ${ }^{7}$ Namely, they contain four oxygen functional groups at positions 2, 3, 9, and 10 on the benzo[c]phenanthridine skeleton. Thereafter, the originally reported structures of both $\mathbf{1}$ and $\mathbf{2}$ were revealed to be incorrect based on synthetic studies. ${ }^{8}$ From the viewpoint of a structure-bioactivity relationship, even if these compounds are not natural products, we considered that the establishment of a synthetic method of such unusual 
benzo[c]phenanthridine derivatives is a meaningful challenge. Thus, in this report, we demonstrate the synthesis of $\mathbf{1}$ and $\mathbf{2}$ using the palladium-mediated intramolecular aryl-aryl coupling reaction as the key step. ${ }^{9}$

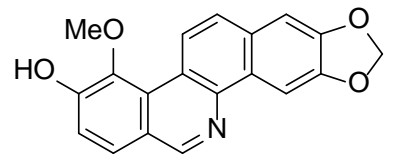

Zanthoxyline (1)

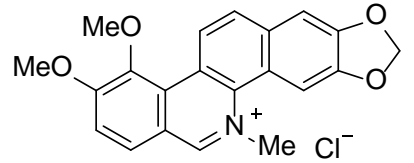

Broussonpapyrine (2)

Figure. Originally Reported Structures of Zanthoxyline (1) and Broussonpapyrine (2)

\section{RESULTS AND DISCUSSION}

For the total synthesis of $\mathbf{1}$, we commenced the preparation of 6,7-methylenedioxy-1-naphthylamine (8) by the reported method (Scheme 1). ${ }^{10}$ First, 2,3-dihydroxynaphtalene (3) was mesylated to afford $\mathbf{4}^{11}$ followed by regioselective nitration for producing $\mathbf{5 .}^{11}$ Alkaline hydrolysis of $\mathbf{5}$ and successive methylenation of the generated catechol moiety produced the tricyclic compound 7 , which was reduced to $\mathbf{8}^{12}$ using the conventional catalytic hydrogenation technique.

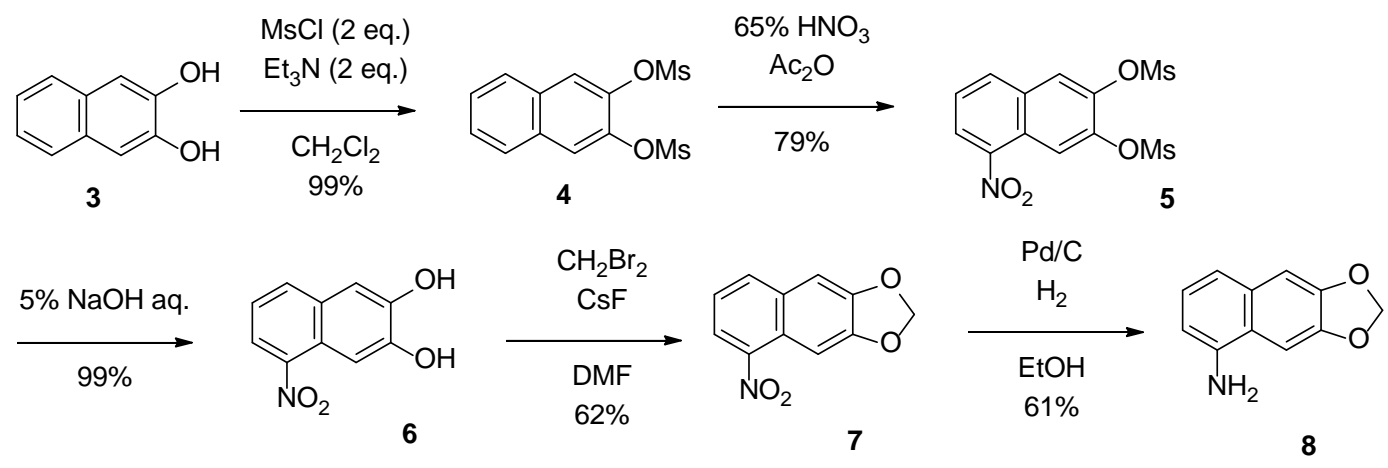

Scheme 1. Preparation of 1-Naphtylamine

Next, we employed 3,4-dihydroxybenzaldehyde (9) as a starting material to prepare the key coupling precursor 15 (Scheme 2). Selective protection of the 4-hydroxy group of $\mathbf{9}$ with the isopropyl unit and successive iodination afforded 11. The leaving hydroxyl group was methylated to form $\mathbf{1 3}$ which was further transformed into the benzoic acid 13. Condensation between $\mathbf{8}$ and $\mathbf{1 3}$ for the amide bond formation using the EDC (1-ethyl-3-(3-dimethylaminopropyl) carbodiimide hydrochloride) - DMAP system produced 14, leading to the $N$-protected compound 15 with the MOM group. The intramolecular aryl-aryl coupling reaction $\left(\mathrm{Pd}(\mathrm{OAc})_{2}-\mathrm{Ag}_{2} \mathrm{CO}_{3}-\mathrm{PPh}_{3}\right)^{4 \mathrm{a}, 13}$ of 15 smoothly proceeded to construct the lactam compound 16. Finally, the synthesis of the target compound $\mathbf{1}$ was completed by the hydride reduction with $\mathrm{LiAlH}_{4}$ and successive acid treatment involving removal of both the MOM and isopropyl groups. 

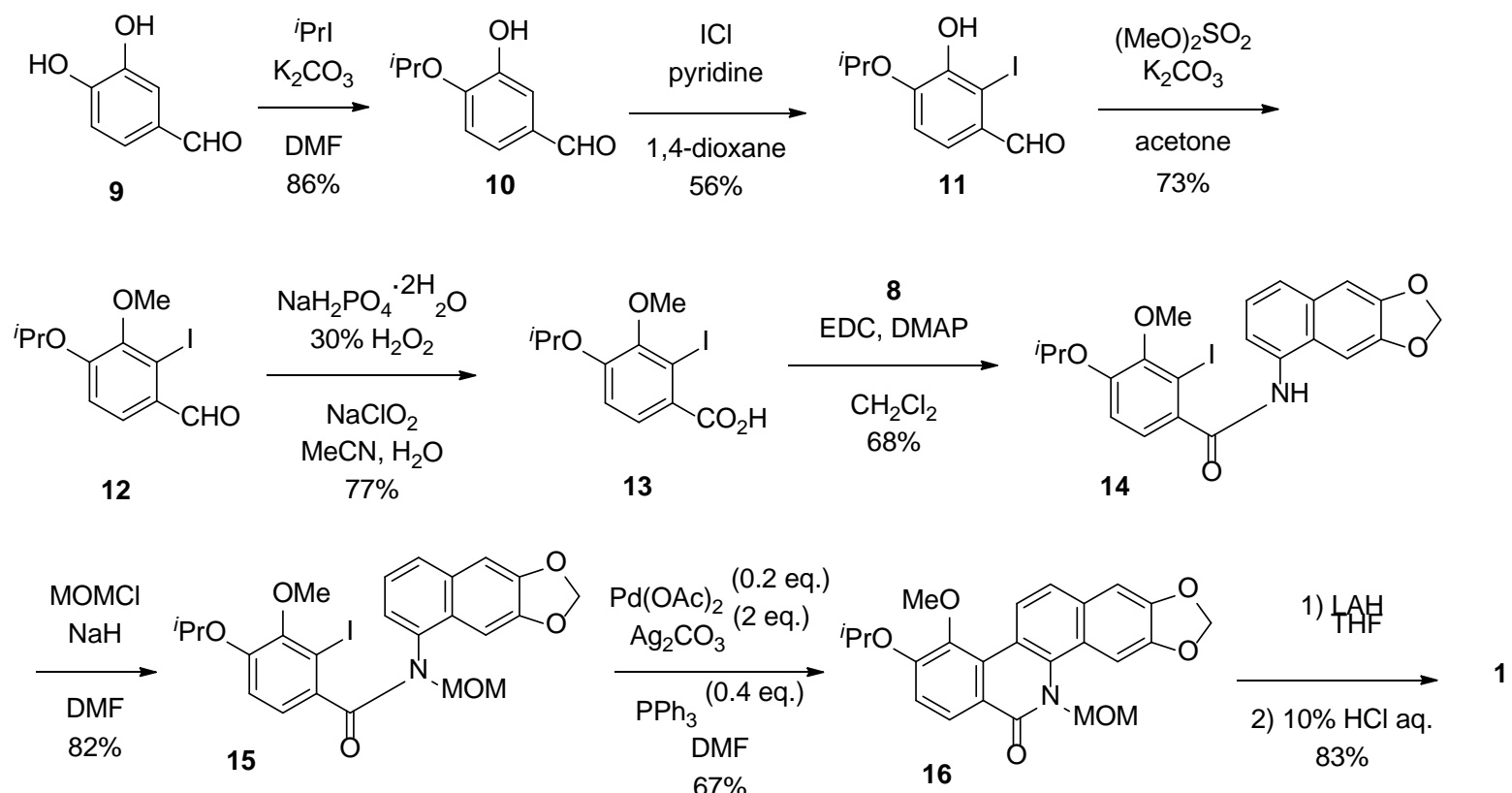

Scheme 2. Synthesis of 1 via Pd-mediated Aryl-Aryl Coupling Reaction

On the other hand, the second target $\mathbf{2}$ was synthesized as illustrated in Scheme 3. Isovanillin $\mathbf{1 7}$ was regioselectively iodinated to prepare $\mathbf{1 8}^{14}$ which was converted into the dimethoxy compound $\mathbf{1 9}^{15}$ using dimethyl sulfate. The formyl group of $\mathbf{1 9}$ was oxidized to a carboxylic acid for the formation of $20{ }^{16}$ which was subjected to the condensation reaction with 8 using EDC-DMAP. Methylation of the amide nitrogen of $\mathbf{2 1}$ was also successful to afford the coupling precursor 22. Conditions similar to Scheme 2 were employed for the intramolecular coupling reaction to produce 23. The final reduction using $\mathrm{LiAlH}_{4}$ followed by the treatment with hydrochloric acid succeeded in the synthesis of $\mathbf{2}$.
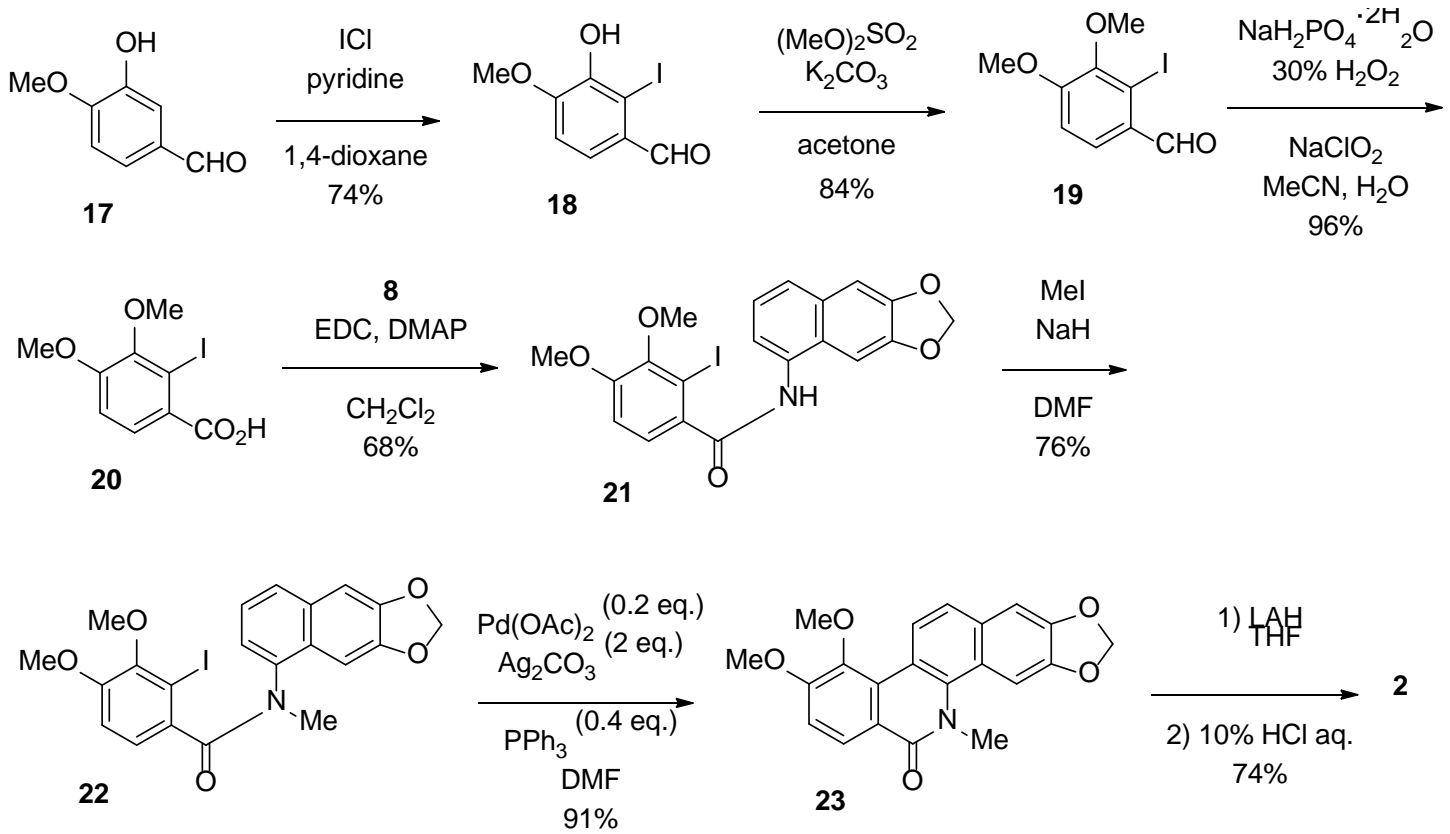


\section{CONCLUSION}

We demonstrated the synthesis of two benzo[c]phenanthridine alkaloids which possess an unusual substituent pattern, i.e., the 2,3,9,10-tetraoxygenated compounds. The Pd-mediated aryl-aryl coupling reaction was efficiently used as the key step for the construction of the benzo[c]phenanthridine skeleton. Further application of this method for the synthesis of other heterocyclic compounds is currently in progress in our laboratory.

\section{EXPERIMENTAL}

General: Melting points were measured using a Yanagimoto micro-melting point hot-plate apparatus and are uncorrected. The IR spectra were recorded using a JASCO FTIR-350 or Shimadzu FTIR-8400 spectrophotometer. The NMR spectra were obtained using a Varian MERCURY-300, JEOL $\alpha-400$, or JNX-ECX500 instrument with the chemical shifts being reported as $\delta$ ppm and the couplings expressed in Hertz. The elemental analysis was performed using a Yanaco MT-5 or Thermo Scientific FlashEA1112 analyzer. Electron ionization mass spectra (EI-MS) was obtained using a JEOL JMS-700 instrument. Silica gel column chromatography was carried out using Merck 9385 Kieselgel 60 or Wako-gel C-200.

\section{2,3-Bis(methylsulfonyloxy)naphthalene (4)}

MsCl (39.3 g, $26.5 \mathrm{ml}, 0.343 \mathrm{~mol}$ ) was added to a solution of 2,3-dihydroxynaphthalene (3) (25 g, 0.156 $\mathrm{mol}), \mathrm{Et}_{3} \mathrm{~N}(34.7 \mathrm{~g}, 47.9 \mathrm{ml}, 0.343 \mathrm{~mol})$ in $\mathrm{CHCl}_{3}(500 \mathrm{ml})$ at $0{ }^{\circ} \mathrm{C}$, then the mixture was allowed to stand at rt. After the resulting precipitates were removed by filtration, water was added to the mother liquid which was extracted with $\mathrm{CHCl}_{3}$. The organic layer was washed with brine, dried oved $\mathrm{MgSO}_{4}$, and concentrated to give a crude residue. Recrystallization from $\mathrm{CHCl}_{3}$ gave 4 (48.8 g, 99\%) in almost a pure form. Colorless needles, mp 153.9-157.2 ${ }^{\circ} \mathrm{C}\left(\mathrm{CHCl}_{3}\right)$ [lit. $\left.{ }^{11} 159-160{ }^{\circ} \mathrm{C}\right] . \mathrm{IR}(\mathrm{KBr}) \mathrm{cm}^{-1}: 1360,1180$. ${ }^{1} \mathrm{H}-\mathrm{NMR}\left(300 \mathrm{MHz}, \mathrm{CDCl}_{3}\right) \delta$ : 3.29 (6H, s, CH 3$), 7.58-7.95$ (6H, m, Ar-H).

\section{6,7-Bis(methylsulfonyloxy)-1-nitronaphthalene (5)}

To a solution of 4 (18 g, $0.057 \mathrm{~mol})$ in acetic anhydride $(180 \mathrm{~mL})$, conc. $\mathrm{HNO}_{3}(42.5 \mathrm{~mL})$ was dropwise added while maintaining the reaction mixture at $35-40{ }^{\circ} \mathrm{C}$. The mixture was then cooled to $5{ }^{\circ} \mathrm{C}$, and stirred for 1 day. After the reaction mixture was poured into ice-water, the resulting precipitates were collected and recrystallized from MeCN to give 5 (16.2 g, 79\%). Yellow needles, 198.8-199.8 ${ }^{\circ} \mathrm{C}$ [lit. ${ }^{11}$ 200-201 ${ }^{\circ} \mathrm{C}$ ]. IR (KBr) cm ${ }^{-1}: 1520,1350,1170 .{ }^{1} \mathrm{H}-\mathrm{NMR}\left(500 \mathrm{MHz}, \mathrm{CDCl}_{3}\right) \delta: 3.37$ (6H, s, $\left.\mathrm{CH}_{3}\right), 7.65$ $\left(1 \mathrm{H}, \mathrm{t}, J=8.0 \mathrm{~Hz}, \mathrm{C}_{7}-\mathrm{H}\right), 8.11\left(1 \mathrm{H}, \mathrm{s}, \mathrm{C}_{1}-\mathrm{H}\right), 8.17\left(1 \mathrm{H}, \mathrm{d}, J=8.5 \mathrm{~Hz}, \mathrm{C}_{8}-\mathrm{H}\right), 8.43\left(1 \mathrm{H}, \mathrm{d}, J=8.5 \mathrm{~Hz} \mathrm{C}_{6}-\mathrm{H}\right)$, $8.78\left(1 \mathrm{H}, \mathrm{s}, \mathrm{C}_{4}-\mathrm{H}\right)$. 


\section{6,7-Dihydroxy-1-nitronaphthalene (6)}

The mixture of 5 (18.7 g, $0.033 \mathrm{~mol})$ and a $5 \% \mathrm{NaOH}$ aqueous solution (165 mL) was heated overnight at $100{ }^{\circ} \mathrm{C}$. After acidification with a $10 \% \mathrm{HCl}$ aqueous solution, the mixture was extracted with AcOEt. The organic layer was washed with brine, dried over $\mathrm{MgSO}_{4}$, and concentrated to give 6 (10.5 g, 99\%). Yellow needles, 206.2-207.0 ${ }^{\circ} \mathrm{C}$ [lit. ${ }^{10} 207-209{ }^{\circ} \mathrm{C}$ ]. IR (KBr) $\mathrm{cm}^{-1}$ : $3360(\mathrm{OH}) .{ }^{1} \mathrm{H}-\mathrm{NMR}(500 \mathrm{MHz}$, $\left.\mathrm{CDCl}_{3}\right) \delta: 5.99(1 \mathrm{H}, \mathrm{s}, \mathrm{ArOH}), 6.31(1 \mathrm{H}, \mathrm{s}, \mathrm{ArOH}), 7.35\left(1 \mathrm{H}, \mathrm{s}, \mathrm{C}_{1}-\mathrm{H}\right), 7.36\left(1 \mathrm{H}, \mathrm{t}, J=8.0 \mathrm{~Hz}, \mathrm{C}_{7}-\mathrm{H}\right)$, $7.92\left(1 \mathrm{H}, \mathrm{d}, J=8.0 \mathrm{~Hz}, \mathrm{C}_{8}-\mathrm{H}\right), 8.15\left(1 \mathrm{H}, \mathrm{s}, \mathrm{C}_{4}-\mathrm{H}\right), 8.17\left(1 \mathrm{H}, \mathrm{d}, J=8.0 \mathrm{~Hz} \mathrm{C}_{6}-\mathrm{H}\right)$.

\section{6,7-Methylenedioxy-1-nitronaphthalene (7)}

CsF (8.51 g, $0.056 \mathrm{~mol}$ ) was added to a solution of 6 (2.3 g, 0.0112mol) in DMF (23 mL), then the mixture was stirred at rt for $1.5 \mathrm{~h}$. To the mixture, $\mathrm{CH}_{2} \mathrm{Br}_{2}$ (2.92 g, 1.18ml, $0.0168 \mathrm{~mol}$ ) was added, which was heated at $115{ }^{\circ} \mathrm{C}$ for $2.5 \mathrm{~h}$. After the mixture was diluted with AcOEt and the insoluble material was removed by filtration, the mixture was washed with a $5 \% \mathrm{NaOH}$ aqueous solution and brine, dried over $\mathrm{MgSO}_{4}$, and concentrated. The resulting solid was recrystallized from AcOEt to give 7 (1.5 g, 62\%). Yellow needles, mp 159.3-163.0 ${ }^{\circ} \mathrm{C}$. IR (KBr) cm ${ }^{-1}: 1470,1320 .{ }^{1} \mathrm{H}-\mathrm{NMR}\left(500 \mathrm{MHz}, \mathrm{CDCl}_{3}\right) \delta: 6.13(2 \mathrm{H}$, s, O-CH $\left.\mathrm{CH}_{2}-\mathrm{O}\right), 7.20\left(1 \mathrm{H}, \mathrm{s}, \mathrm{C}_{1}-\mathrm{H}\right), 7.38\left(1 \mathrm{H}, \mathrm{t}, J=8.0 \mathrm{~Hz}, \mathrm{C}_{7}-\mathrm{H}\right), 7.91$ (1H, d, $\left.J=7.5 \mathrm{~Hz}, \mathrm{C}_{8}-\mathrm{H}\right), 7.98(1 \mathrm{H}$, s, $\left.\mathrm{C}_{4}-\mathrm{H}\right), 8.17\left(1 \mathrm{H}, \mathrm{d}, J=7.5 \mathrm{~Hz} \mathrm{C}_{6}-\mathrm{H}\right)$.

\section{1-Amino-6,7-methylenedioxynaphthalene (8)}

Under an $\mathrm{H}_{2}$ atmosphere, the mixture of 7 (2.0 g, $\left.0.0092 \mathrm{~mol}\right)$, EtOH $(100 \mathrm{~mL})$, and 10\% Pd/C (250 mg) was vigorously stirred at $\mathrm{rt}$ for $1.5 \mathrm{~h}$. After filtration, the solvent was evaporated to give a crude residue, and then recrystallization from EtOH gave 8 (1.06 g, 61\%). Yellow needles, mp 151.5-154.0 ${ }^{\circ} \mathrm{C}$ [lit. ${ }^{12 \mathrm{a}}$ 154-155 ${ }^{\circ} \mathrm{C}$ (Et $\left.\left.{ }_{2} \mathrm{O}-h e x a n e\right) ; ~ l i t .{ }^{12 \mathrm{~b}} 152-154{ }^{\circ} \mathrm{C}\left(\mathrm{EtOH}-\mathrm{H}_{2} \mathrm{O}\right)\right]$. IR $(\mathrm{KBr}) \mathrm{cm}^{-1}: 3210(\mathrm{NH}) .{ }^{1} \mathrm{H}-\mathrm{NMR}$ $\left(500 \mathrm{MHz}, \mathrm{CDCl}_{3}\right) \delta: 6.03\left(2 \mathrm{H}, \mathrm{s}, \mathrm{O}-\mathrm{CH}_{2}-\mathrm{O}\right), 6.29-7.21$ (5H, m, Ar-H).

\section{3-Hydroxy-4-isopropoxybenzaldehyde (10)}

To a suspension of $\mathrm{K}_{2} \mathrm{CO}_{3}(12.5 \mathrm{~g}, 0.090 \mathrm{~mol})$ in DMF (50 mL), 3,4-dihydroxybenzaldehyde (9, 25 g, $0.28 \mathrm{~mol})$ and 2-iodopropane $(18 \mathrm{~mL}, 0.18 \mathrm{~mol})$ were successively added, then the mixture was stirred at $50{ }^{\circ} \mathrm{C}$ for $1 \mathrm{~d}$. The mixture was acidified with a $10 \% \mathrm{HCl}$ aqueous solution and extracted with AcOEt. The organic layer was washed with brine, dried over $\mathrm{MgSO}_{4}$, and concentrated to give a residue which was subjected to silica gel column chromatography with AcOEt-Hexane (1:6). The title compound 10 (28.2 g, 86 \%) was obtained. Yellow needles, mp 63.7-65.2 ${ }^{\circ} \mathrm{C}$ (AcOEt). IR (KBr) cm ${ }^{-1}: 3300(\mathrm{OH}), 1680$ $(\mathrm{C}=\mathrm{O}), 1280 .{ }^{1} \mathrm{H}-\mathrm{NMR}\left(300 \mathrm{MHz}, \mathrm{CDCl}_{3}\right) \delta: 1.41$ (3H, s, $\left.\mathrm{ArOCHCH}_{3}\right), 1.42\left(3 \mathrm{H}, \mathrm{s}, \mathrm{ArOCHCH}_{3}\right), 4.72$ $\left(1 \mathrm{H}\right.$, heptet, $\left.J=6.3 \mathrm{~Hz}, \operatorname{ArOCH}\left(\mathrm{CH}_{3}\right)_{2}\right), 5.89(1 \mathrm{H}, \mathrm{s}, \mathrm{ArOH}), 6.95\left(1 \mathrm{H}, \mathrm{d}, J=8.0 \mathrm{~Hz}, \mathrm{C}_{5}-\mathrm{H}\right), 7.39(1 \mathrm{H}$, dd, 
$\left.J=8.0,2.5 \mathrm{~Hz}, \mathrm{C}_{6}-\mathrm{H}\right), 7.43\left(1 \mathrm{H}, \mathrm{d}, J=2.5 \mathrm{~Hz}, \mathrm{C}_{2}-\mathrm{H}\right), 9.82\left(1 \mathrm{H}, \mathrm{s}\right.$, ArCHO). Anal. Calcd for $\mathrm{C}_{10} \mathrm{H}_{12} \mathrm{O}_{3}: \mathrm{C}_{\text {, }}$ 66.65; H, 6.71. Found: C, 66.61; H, 6.52.

\section{3-Hydroxy-2-iodo-4-isopropoxybenzaldehyde (11)}

To a solution of $10(12 \mathrm{~g}, 0.13 \mathrm{~mol})$ in pyridine $(80 \mathrm{~mL})$, a solution of ICl $(21.9 \mathrm{~g}, 0.135 \mathrm{~mol})$ in 1,4-dioxane $(160 \mathrm{~mL})$ was dropwise added under cooling at $0{ }^{\circ} \mathrm{C}$. The mixture was warmed to $\mathrm{rt}$ and allowed to stand for $15 \mathrm{~h}$. After the volatile materials were removed in vacuo, water was added to the mixture. The resulting precipitates were collected, then recrystallization from AcOEt gave 11 (14.27 g, 56\%). Pale yellow needles, mp 152.5-156.7 ${ }^{\circ} \mathrm{C}$ (AcOEt). IR (KBr) cm ${ }^{-1}: 3400$ (OH), 1670 (CO), 1280. ${ }^{1} \mathrm{H}-\mathrm{NMR}\left(300 \mathrm{MHz}, \mathrm{CDCl}_{3}\right.$ ) $\delta: 1.41$ (3H, s, $\left.\mathrm{ArOCHCH}_{3}\right), 1.43$ (3H, s, ArOCHCH 3$), 4.75$ (1H, heptet, $J=$ $\left.6.3 \mathrm{~Hz}, \operatorname{ArOCH}\left(\mathrm{CH}_{3}\right)_{2}\right), 6.36(1 \mathrm{H}, \mathrm{s}, \mathrm{ArOH}), 6.89\left(1 \mathrm{H}, \mathrm{d}, J=8.7 \mathrm{~Hz}, \mathrm{C}_{5}-\mathrm{H}\right), 7.53(1 \mathrm{H}, \mathrm{d}, J=8.7 \mathrm{~Hz}$, $\left.\mathrm{C}_{6}-\mathrm{H}\right), 10.03(1 \mathrm{H}, \mathrm{s}, \mathrm{ArCHO}) .{ }^{13} \mathrm{C}-\mathrm{NMR}\left(125 \mathrm{MHz} \mathrm{CDCl}_{3}\right) \delta: 194.9,149.0,146.4,128.4,123.6,111.6$, 88.2, 72.7, 22.1. Anal. Calcd for $\mathrm{C}_{10} \mathrm{H}_{11} \mathrm{IO}_{3}$ : C, 39.24; H, 3.62. Found: C, 39.23; H, 3.64.

\section{2-Iodo-4-isopropoxy-3-methoxybenzaldehyde (12)}

A mixture of 11 (14 g, $0.045 \mathrm{~mol}), \mathrm{K}_{2} \mathrm{CO}_{3}(12.7 \mathrm{~g}, 0.092 \mathrm{~mol}),(\mathrm{MeO})_{2} \mathrm{SO}_{2}(8.66 \mathrm{~mL}, 0.092 \mathrm{~mol})$, and acetone $(50 \mathrm{~mL})$ was heated under reflux for $1 \mathrm{~h}$. The solvent was removed under reduced pressure, then water was added to the mixture which was extracted with ether. The organic layer was washed with brine, dried over $\mathrm{MgSO}_{4}$, and concentrated to give a crude solid. Recrystallization from ether gave 12 (10.6 g, 73\%). Pale yellow prisms, mp 48.1-51.9 ${ }^{\circ} \mathrm{C}\left(\mathrm{Et}_{2} \mathrm{O}\right) .{ }^{1} \mathrm{H}-\mathrm{NMR}\left(500 \mathrm{MHz}, \mathrm{CDCl}_{3}\right) \delta: 1.41(3 \mathrm{H}, \mathrm{s}$, $\left.\mathrm{ArOCHCH}_{3}\right), 1.42\left(3 \mathrm{H}, \mathrm{s}, \mathrm{ArOCHCH}_{3}\right), 3.86\left(3 \mathrm{H}, \mathrm{s}, \mathrm{ArOCH}_{3}\right), 4.70$ (1H, heptet, $\left.J=6.0 \mathrm{~Hz}, \mathrm{ArOCH}\right)$, $6.94\left(1 \mathrm{H}, \mathrm{d}, J=8.5 \mathrm{~Hz}, \mathrm{C}_{5}-\mathrm{H}\right), 7.68\left(1 \mathrm{H}, \mathrm{d}, J=8.5 \mathrm{~Hz}, \mathrm{C}_{6}-\mathrm{H}\right) .{ }^{13} \mathrm{C}-\mathrm{NMR}\left(125 \mathrm{MHz}, \mathrm{CDCl}_{3}\right) \delta: 195.1$, 156.2, 149.4, 128.6, 127.2, 113.8, 100.9, 71.7, 60.3, 22.0. Anal. Calcd for $\mathrm{C}_{11} \mathrm{H}_{13} \mathrm{IO}_{3}$ : C, 41.27; H, 4.09. Found: C, 41.50; H, 4.07.

\section{2-Iodo-4-isopropoxy-3-methoxybenzoic acid (13)}

To a solution of 12 (150 mg, $0.467 \mathrm{mmol}), \mathrm{NaH}_{2} \mathrm{PO}_{4} \cdot 2 \mathrm{H}_{2} \mathrm{O}$ (18.3 mg, $\left.0.12 \mathrm{mmol}\right)$, and $31 \% \mathrm{H}_{2} \mathrm{O}_{2}(79.7$ mg, $2.34 \mathrm{mmol})$ in $\mathrm{MeCN}(15 \mathrm{~mL})$, an 80\% aqueous solution of $\mathrm{NaClO}_{2}(0.5 \mathrm{~mL}, 79.5 \mathrm{mg}, 0.879 \mathrm{mmol})$ was added at $0{ }^{\circ} \mathrm{C}$. The mixture was stirred at the same temperature for $5.5 \mathrm{~h}$, then poured into a sat. $\mathrm{NaHSO}_{3}$ aqueous solution. After acidification with $10 \% \mathrm{HCl}$ aqueous solution, the mixture was extracted with AcOEt. The organic layer was washed with brine, dried over $\mathrm{MgSO}_{4}$, and concentrated to give a residue. Recrystallization from AcOEt gave 13 (120 mg, 77\%). Colorless needles, mp 152-153 ${ }^{\circ} \mathrm{C}$ (AcOEt). IR (KBr) cm ${ }^{-1}: 3000(\mathrm{OH}), 1700(\mathrm{CO}), 1280 .{ }^{1} \mathrm{H}-\mathrm{NMR}\left(300 \mathrm{MHz}, \mathrm{CDCl}_{3}\right)$ \&: $1.40(3 \mathrm{H}, \mathrm{s}$, $\left.\mathrm{ArOCHCH}_{3}\right), 1.42\left(3 \mathrm{H}, \mathrm{s}, \mathrm{ArOCHCH}_{3}\right), 3.84\left(3 \mathrm{H}, \mathrm{s}, \mathrm{ArOCH}_{3}\right), 4.67(1 \mathrm{H}$, heptet, $J=6.3 \mathrm{~Hz}$, 
$\left.\operatorname{ArOCH}\left(\mathrm{CH}_{3}\right)_{2}\right), 6.90\left(1 \mathrm{H}, \mathrm{d}, J=8.7 \mathrm{~Hz}, \mathrm{C}_{5}-\mathrm{H}\right), 7.82\left(1 \mathrm{H}, \mathrm{d}, J=8.7 \mathrm{~Hz}, \mathrm{C}_{6}-\mathrm{H}\right) .{ }^{13} \mathrm{C}-\mathrm{NMR}(125 \mathrm{MHz}$, $\left.\mathrm{CDCl}_{3}\right) \delta: 171.5,154.5,150.8,129.4,125.7,113.7,95.9,71.7,60.3$, 22.1. Anal. Calcd for $\mathrm{C}_{11} \mathrm{H}_{13} \mathrm{IO}_{4}: \mathrm{C}_{\text {, }}$ 39.31; H, 3.90. Found: C, 39.27; H, 3.86.

\section{2-Iodo-4-isopropoxy-3-methoxy- $N$-(6,7-methylenedioxy-1-naphthyl)benzamide (14)}

A mixture of 13 (100 mg, $0.31 \mathrm{mmol}), 8$ (67 mg, $0.37 \mathrm{mmol})$, EDC (98 mg, $0.53 \mathrm{mmol})$, DMAP (7.9 mg, $0.068 \mathrm{mmol})$, and $\mathrm{CH}_{2} \mathrm{Cl}_{2}(10 \mathrm{~mL})$ was heated at $30{ }^{\circ} \mathrm{C}$ for $4.5 \mathrm{~h}$. After being poured into water, the mixture was extracted with $\mathrm{CH}_{2} \mathrm{Cl}_{2}$. The organic layer was washed with brine, dried over $\mathrm{MgSO}_{4}$, and concentrated to give a residue which was subjected to silica gel column chromatography with AcOEt/hexane (1:4). Recrystallization from $\mathrm{CH}_{2} \mathrm{Cl}_{2}$ gave 14 (103 mg, 68\%). Colorless needles, mp 226-229 ${ }^{\circ} \mathrm{C}\left(\mathrm{CH}_{2} \mathrm{Cl}_{2}\right) .{ }^{1} \mathrm{H}-\mathrm{NMR}\left(300 \mathrm{MHz}, \mathrm{CDCl}_{3}\right) \delta: 1.40$ (3H, s, $\left.\mathrm{ArOCHCH}_{3}\right), 1.42$ (3H, s, $\left.\mathrm{ArOCHCH}_{3}\right)$, 3.89 (3H, s, $\left.\mathrm{ArOCH}_{3}\right), 4.65$ (1H, heptet, $\left.J=6.3 \mathrm{~Hz}, \operatorname{ArOCH}\left(\mathrm{CH}_{3}\right)_{2}\right), 6.05\left(2 \mathrm{H}, \mathrm{s}, \mathrm{OCH}_{2} \mathrm{O}\right), 6.98(1 \mathrm{H}, \mathrm{d}, J$ $\left.=8.1 \mathrm{~Hz}, \mathrm{C}_{5}-\mathrm{H}\right), 7.33-7.61(5 \mathrm{H}, \mathrm{m}, \mathrm{Ar}-\mathrm{H}), 7.83\left(1 \mathrm{H}, \mathrm{d}, J=8.1 \mathrm{~Hz}, \mathrm{C}_{6}-\mathrm{H}\right) .{ }^{13} \mathrm{C}-\mathrm{NMR}\left(125 \mathrm{MHz}, \mathrm{CDCl}_{3}\right)$ $\delta: 167.9,152.2,150.3,148.5,147.8,135.5,135.4,131.6,125.9,125.3,124.9,124.4,121.1,115.5,104.7$, 101.4, 98.5, 92.5, 71.7, 60.4, 22.1. Anal. Calcd for $\mathrm{C}_{22} \mathrm{H}_{20} \mathrm{INO}_{5}$ : C, 52.29; H, 3.99; N, 2.77. Found: C, 52.38; H, 4.10; N, 2.69.

\section{2-Iodo-4-isopropoxy-3-methoxy- $N$-(methoxymethyl)- $N$-(6,7-methylenedioxy-1-naphthyl)benzamide} (15)

$\mathrm{NaH}$ (50\% in mineral oil, $28 \mathrm{mg}, 0.59 \mathrm{mmol}$ ) was washed with hexane before use. To a mixture of the prepared base and DMF (10 mL), 14 (100 mg, $0.198 \mathrm{mmol})$ was added and stirred at rt for $30 \mathrm{~min}$. MOMCl (24 mg, $0.023 \mathrm{ml}, 0.30 \mathrm{mmol}$ ) was added to the mixture, then stirred at rt for $5 \mathrm{~h}$. The mixture was poured into water and extracted with AcOEt. The organic layer was washed with brine, dried over $\mathrm{MgSO}_{4}$, and concentrated to give a residue. Silica gel column chromatography with AcOEt/Hexane (1:3) gave 15 (88.7 mg, 82\%) as an amorphous solid. ${ }^{1} \mathrm{H}-\mathrm{NMR}\left(300 \mathrm{MHz}, \mathrm{CDCl}_{3}\right) \delta$ : 1.17-1.42 (6H, m, $\left.\operatorname{ArOCH}\left(\mathrm{CH}_{3}\right)_{2}\right)$, 3.69-3.89 (6H, m, N-CH $\left.\mathrm{OCH}_{3}, \mathrm{ArOCH}_{3}\right)$, 4.29-4.68 (1H, m, $\left.\operatorname{ArOCH}\left(\mathrm{CH}_{3}\right)_{2}\right)$, 4.86, 5.75 (2H, d, $\left.J=9.9, \mathrm{NCH}_{2} \mathrm{OCH}_{3}\right), 6.32-6.37\left(1 \mathrm{H}, \mathrm{m}, \mathrm{C}_{5},-\mathrm{H}\right), 6.56-6.64\left(1 \mathrm{H}, \mathrm{m}, \mathrm{C}_{6},-\mathrm{H}\right), 7.06-7.50$ (5H, m, $\mathrm{Ar}-\mathrm{H}) .{ }^{13} \mathrm{C}-\mathrm{NMR}\left(125 \mathrm{MHz}, \mathrm{CDCl}_{3}\right.$ ) (selected peaks) $\delta: 171.6,150.5,150.1,148.9,147.9,136.7,131.6$, 127.7, 126.2, 124.1, 114.4, 104.6, 101.5, 99.3, 93.4, 78.7, 71.3, 60.2, 57.9, 22.1, 21.7. Anal. Calcd for $\mathrm{C}_{24} \mathrm{H}_{24} \mathrm{INO}_{6}$ : C, 52.47; H, 4.40; N, 2.55. Found: C, 52.71; H, 4.51: N, 2.37.

\section{9-Isopropoxy-10-methoxy- $N$-methoxymethyl-2,3-methylenedioxybenzo[c]phenanthridine-6(5H)- one (16)}

A mixture of 15 (100 mg, $0.18 \mathrm{mmol}), \mathrm{PPh}_{3}$ (19.2 mg, $\left.0.072 \mathrm{mmol}\right), \mathrm{Ag}_{2} \mathrm{CO}_{3}$ (101 mg, $\left.0.36 \mathrm{mmol}\right)$ 
$\mathrm{Pd}(\mathrm{OAc})_{2}$ (8.2 mg, $\left.0.036 \mathrm{mmol}\right)$, and DMF (2 mL) was heated under reflux for $1 \mathrm{~h}$. After the mixture was diluted with AcOEt, any undissolved materials were filtered off. The mother liquid was washed with brine, dried over $\mathrm{MgSO}_{4}$, and concentrated to give a residue which was subjected to silica gel column chromatography with AcOEt-hexane (1:4). The title compound 16 (52 mg, 67\%) was obtained, which was recrystallized from AcOEt for use as an analytical sample. Yellow needles, mp 175.7-177.7 ${ }^{\circ} \mathrm{C}$ (AcOEt). IR (KBr) cm ${ }^{-1}: 1660$ (CO). ${ }^{1} \mathrm{H}-\mathrm{NMR}\left(300 \mathrm{MHz}, \mathrm{CDCl}_{3}\right) \delta: 1.46$ (3H, s, $\left.\mathrm{ArOCHCH}_{3}\right), 1.48$ (3H, s, $\left.\operatorname{ArOCHCH}_{3}\right), 3.72\left(3 \mathrm{H}, \mathrm{s}, \mathrm{NCH}_{2} \mathrm{OCH}_{3}\right), 3.87\left(3 \mathrm{H}, \mathrm{s}, \mathrm{ArOCH}_{3}\right), 4.77(1 \mathrm{H}$, heptet, $J=6.3 \mathrm{~Hz}$, $\left.\operatorname{ArOCH}\left(\mathrm{CH}_{3}\right)_{2}\right), 5.37\left(2 \mathrm{H}, \mathrm{s}, \mathrm{NCH}_{2} \mathrm{OCH}_{3}\right), 6.09$ (2H, s, $\left.\mathrm{OCH}_{2} \mathrm{O}\right), 7.15-7.56(4 \mathrm{H}, \mathrm{m}, \mathrm{Ar}-\mathrm{H}), 8.35(1 \mathrm{H}, \mathrm{d}, J$ = 9.0, $\left.\mathrm{C}_{7}-\mathrm{H}\right), 9.12\left(1 \mathrm{H}, \mathrm{d}, J=9.0, \mathrm{C}_{8}-\mathrm{H}\right) .{ }^{13} \mathrm{C}-\mathrm{NMR}\left(75 \mathrm{MHz}, \mathrm{CDCl}_{3}\right) \delta: 165.1,155.6,147.8,147.6,146.3$, 136.3, 132.0, 128.7, 125.8, 123.2, 122.9, 121.2, 119.5, 116.7, 114.6, 104.0, 102.7, 101.3, 82.1, 71.1, 60.0, 57.1, 22.1. Anal. Calcd for $\mathrm{C}_{24} \mathrm{H}_{23} \mathrm{NO}_{6} \cdot 0.5 \mathrm{H}_{2} \mathrm{O}$ : C, 66.97; H, 5.62; N, 3.25. Found C, 66.93, H, 5.56; N, 3.34 .

\section{9-Hydroxy-10-methoxy-2,3-methylenedioxybenzo[c]phenanthridine (1)}

To a solution of 16 (120 mg, $0.285 \mathrm{mmol})$ in THF (5 ml), $\mathrm{LiAlH}_{4}(32 \mathrm{mg}, 0.855 \mathrm{mmol})$ was added at $0{ }^{\circ} \mathrm{C}$, then the mixture was vigorously stirred for $1 \mathrm{~h}$. After quenching with ice water, an $\mathrm{NaOH}$ aqueous solution was added as the mixture was adjusted to $\mathrm{pH} 12-13$. After extraction with $\mathrm{Et}_{2} \mathrm{O}$, the organic layer was washed with brine, dried over $\mathrm{K}_{2} \mathrm{CO}_{3}$, and concentrated. To the resulting solid, a conc. $\mathrm{HCl}$ aqueous solution (10 mL) was added, then the mixture was stirred for $24 \mathrm{~h}$. After adjusting to $\mathrm{pH} 9$ by adding a sat. $\mathrm{NH}_{4} \mathrm{Cl}$ aqueous solution, the mixture was extracted with AcOEt. The organic layer was washed with brine, dried over $\mathrm{Na}_{2} \mathrm{SO}_{4}$, and concentrated to give a residue which was subjected to silica gel column chromatography with AcOEt-hexane (1:2). The title compound 1 (63 mg, $83 \%$ ) was obtained, which was recrystallized from $\mathrm{CHCl}_{3}$ for use as an analytical sample. Brown needles, mp 226-228 ${ }^{\circ} \mathrm{C}\left(\mathrm{CHCl}_{3}\right)$ [lit. ${ }^{5}$ 220-222 ${ }^{\circ} \mathrm{C}$ (originally reported zanthoxyline); lit. ${ }^{8 \mathrm{~b}} 226-227^{\circ} \mathrm{C}$ (synthesized by Hibino)]. IR (KBr) $\mathrm{cm}^{-1}$ : $3400(\mathrm{OH}), 1480 .{ }^{1} \mathrm{H}-\mathrm{NMR}\left(300 \mathrm{MHz}, \mathrm{DMSO}_{-} \mathrm{d}_{6}\right) \delta: 3.88\left(3 \mathrm{H}, \mathrm{s}, \mathrm{ArOCH}_{3}\right), 6.21\left(2 \mathrm{H}, \mathrm{s}, \mathrm{OCH}_{2} \mathrm{O}\right), 7.43$ $\left(1 \mathrm{H}, \mathrm{d}, J=9.0 \mathrm{~Hz}, \mathrm{C}_{8}-\mathrm{H}\right), 7.48\left(1 \mathrm{H}, \mathrm{s}, \mathrm{C}_{1}-\mathrm{H}\right), 7.94\left(2 \mathrm{H}, \mathrm{d}, J=9.0 \mathrm{~Hz}, \mathrm{C}_{7,12}-\mathrm{H}\right), 8.58\left(1 \mathrm{H}, \mathrm{s}, \mathrm{C}_{4}-\mathrm{H}\right), 9.21$ $\left(1 \mathrm{H}, \mathrm{d}, J=9.0 \mathrm{~Hz}, \mathrm{C}_{11}-\mathrm{H}\right), 9.27\left(1 \mathrm{H}, \mathrm{s}, \mathrm{C}_{6}-\mathrm{H}\right), 10.46(1 \mathrm{H}, \mathrm{br}, \mathrm{OH}) .{ }^{13} \mathrm{C}-\mathrm{NMR}\left(75 \mathrm{MHz}, \mathrm{DMSO}-\mathrm{d}_{6}\right) \delta$ : 153.43, 151.88, 148.52, 148.10, 143.05, 129.86, 127.03, 126.57, 126.36, 122.12, 119.27, 119.14, 104.16, 101.72, 101.66, 59.65. Anal. Calcd for $\mathrm{C}_{19} \mathrm{H}_{13} \mathrm{NO}_{4} \cdot 0.5 \mathrm{H}_{2} \mathrm{O}$ : C, 69.51; H, 4.30; N, 4.27. Found: C, 69.70; H, 4.26; N, 4.26. FAB-MS m/z: $320(\mathrm{M}+1)^{+}$.

\section{3-Hydroxy-2-iodo-4-methoxybenzaldehyde (18)}

To a solution of isovanillin (17) $(10.0 \mathrm{~g}, 65.7 \mathrm{mmol})$ in pyridine $(40 \mathrm{~mL})$, a solution of ICl $(12.1 \mathrm{~g}, 74.5$ mmol) in 1,4-dioxane ( $80 \mathrm{~mL}$ ) was dropwise added under cooling at $0^{\circ} \mathrm{C}$. The mixture was warmed to rt 
and allowed to stand for $3 \mathrm{~h}$. After water was added to the mixture, the resulting precipitates were collected, then recrystallization from AcOEt gave 18 (13.4 g, 74\%). Yellow needles, mp 162.3-163.6 ${ }^{\circ} \mathrm{C}$ (AcOEt) [lit. ${ }^{14} 169-171.5^{\circ} \mathrm{C}$ ]. ${ }^{1} \mathrm{H}-\mathrm{NMR}\left(500 \mathrm{MHz}, \mathrm{CDCl}_{3}\right) \delta: 10.03$ (1H, s, ArCHO), 7.56 (1H, d, $J=8.3$ $\left.\mathrm{Hz}, \mathrm{C}_{5}-\mathrm{H}\right), 6.93\left(1 \mathrm{H}, \mathrm{d}, J=8.3 \mathrm{~Hz}, \mathrm{C}_{6}-\mathrm{H}\right), 6.31(1 \mathrm{H}, \mathrm{s}, \mathrm{ArOH}), 4.00\left(3 \mathrm{H}, \mathrm{s}, \mathrm{ArOCH}_{3}\right)$.

\section{2-Iodo-3,4-dimethoxybenzaldehyde (19)}

A mixture of 11 (10.0 g, $36.0 \mathrm{mmol}), \mathrm{K}_{2} \mathrm{CO}_{3}(9.40 \mathrm{~g}, 68.0 \mathrm{mmol}),(\mathrm{MeO})_{2} \mathrm{SO}_{2}(6.80 \mathrm{~mL}, 70.1 \mathrm{mmol})$, and acetone $(50 \mathrm{~mL})$ was heated under reflux for $1 \mathrm{~h}$. The solvent was removed under reduced pressure, and then water was added to the mixture which was extracted with ether. The organic layer was washed with brine, dried over $\mathrm{MgSO}_{4}$, and concentrated to give a crude solid. Recrystallization from ether gave 19 (8.78 g, 84\%). Colorless needles, mp 80.5-82.0 ${ }^{\circ} \mathrm{C}$ (AcOEt) [lit. ${ }^{15 a} 82{ }^{\circ} \mathrm{C}$ ]. ${ }^{1} \mathrm{H}-\mathrm{NMR}\left(500 \mathrm{MHz}, \mathrm{CDCl}_{3}\right) \delta$ : $9.93(1 \mathrm{H}, \mathrm{s}, \operatorname{ArCHO}), 7.63\left(1 \mathrm{H}, \mathrm{d}, J=8.5 \mathrm{~Hz}, \mathrm{C}_{6}-\mathrm{H}\right), 6.92\left(1 \mathrm{H}, \mathrm{d}, J=8.5 \mathrm{~Hz}, \mathrm{C}_{5}-\mathrm{H}\right), 3.91(3 \mathrm{H}, \mathrm{s}$, $\left.\mathrm{ArOCH}_{3}\right), 3.80\left(3 \mathrm{H}, \mathrm{s}, \mathrm{ArOCH}_{3}\right)$.

\section{2-Iodo-3,4-dimethoxybenzoic acid (20)}

To a solution of 19 (1.00 g, $3.42 \mathrm{mmol}), \mathrm{NaH}_{2} \mathrm{PO}_{4} \cdot 2 \mathrm{H}_{2} \mathrm{O}$ (137 mg, $\left.0.878 \mathrm{mmol}\right)$, and $30 \% \mathrm{H}_{2} \mathrm{O}_{2}$ (600 mg, $5.29 \mathrm{mmol})$ in $\mathrm{MeCN}(15 \mathrm{~mL})$, an $80 \%$ aqueous solution of $\mathrm{NaClO}_{2}(1.5 \mathrm{~mL}, 385 \mathrm{mg}, 5.34 \mathrm{mmol})$ was added at $0{ }^{\circ} \mathrm{C}$. The mixture was stirred at the same temperature for $4 \mathrm{~h}$, then poured into a $10 \% \mathrm{Na}_{2} \mathrm{~S}_{2} \mathrm{O}_{3}$ aqueous solution. After acidification with a $10 \% \mathrm{HCl}$ aqueous solution, the mixture was extracted with AcOEt. The organic layer was washed with brine, dried over $\mathrm{MgSO}_{4}$, and concentrated to give a residue. Recrystallization from AcOEt gave 20 (1.01 g, 96\%). Colorless needles, mp 203.8-204.5 ${ }^{\circ} \mathrm{C}$ (AcOEt) [lit. ${ }^{16}$ 204.5-205 ${ }^{\circ} \mathrm{C}$ ]. ${ }^{1} \mathrm{H}-\mathrm{NMR}\left(500 \mathrm{MHz}, \mathrm{CDCl}_{3}\right) \delta: 7.85\left(1 \mathrm{H}, \mathrm{d}, J=8.5 \mathrm{~Hz}, \mathrm{C}_{6}-\mathrm{H}\right), 6.93(1 \mathrm{H}, \mathrm{d}, J=8.5$ $\left.\mathrm{Hz}, \mathrm{C}_{5}-\mathrm{H}\right), 3.94$ (3H, s, $\left.\mathrm{ArOCH}_{3}\right), 3.85$ (3H, s, $\left.\mathrm{ArOCH}_{3}\right)$.

\section{2-Iodo-3,4-dimethoxy- $N$-(6,7-methylenedioxy-1-naphthyl)benzamide (21)}

A mixture of 20 (197 mg, $0.641 \mathrm{mmol}), 8$ (100 mg, $0.534 \mathrm{mmol}$ ), EDC (246 mg, $1.28 \mathrm{mmol})$, DMAP (7.3 mg, $0.059 \mathrm{mmol})$, and $\mathrm{CH}_{2} \mathrm{Cl}_{2}(10 \mathrm{~mL})$ was stirred at rt overnight. After being poured into water, the mixture was extracted with $\mathrm{CH}_{2} \mathrm{Cl}_{2}$. The organic layer was washed with brine, dried over $\mathrm{MgSO}_{4}$, and concentrated to give a residue which was subjected to silica gel column chromatography with AcOEt/hexane (1:4). Recrystallization from $\mathrm{CHCl}_{3}-\mathrm{MeOH}$ gave 21 (173 mg, 68\%). Colorless needles, mp 124.6-125.8 ${ }^{\circ} \mathrm{C}\left(\mathrm{CHCl}_{3}-\mathrm{MeOH}\right)$. IR (KBr) cm ${ }^{-1}$ : 1654 (CO), 1468 (C-N), 1289, 1250 (COC). ${ }^{1} \mathrm{H}-\mathrm{NMR}\left(500 \mathrm{MHz}, \mathrm{DMSO}_{6}\right.$ ) $\delta: 10.18(1 \mathrm{H}, \mathrm{s}), 7.65\left(1 \mathrm{H}, \mathrm{d}, J=8.3 \mathrm{~Hz}, \mathrm{C}_{6}-\mathrm{H}\right), 7.54(1 \mathrm{H}, \mathrm{d}, J=7.5 \mathrm{~Hz}$, naphC $\left._{2}-\mathrm{H}\right), 7.49$ (1H, s, naphC $\left.5-\mathrm{H}\right), 7.31$ (3H, m, naphC $\left.2, \mathrm{C}_{4}, \mathrm{C}_{8}-\mathrm{H}\right), 7.19\left(1 \mathrm{H}, \mathrm{d}, J=8.3 \mathrm{~Hz}, \mathrm{C}_{5}-\mathrm{H}\right), 6.14$ $\left(2 \mathrm{H}, \mathrm{s}, \mathrm{OCH}_{2} \mathrm{O}\right), 3.88\left(3 \mathrm{H}, \mathrm{s}, \mathrm{OCH}_{3}\right), 3.76\left(1 \mathrm{H}, \mathrm{s}, \mathrm{OCH}_{3}\right) .{ }^{13} \mathrm{C}-\mathrm{NMR}\left(125 \mathrm{MHz}, \mathrm{DMSO}-\mathrm{d}_{6}\right) \delta:$ 168.3, 
153.0, 148.4, 147.7, 147.5, 136.7, 133.0, 131.3, 125.8, 125.3, 124.4, 124.0, 121.7, 112.9, 104.0, 101.5, 99.9, 93.0, 60.0, 56.4. Anal. Calcd for $\mathrm{C}_{20} \mathrm{H}_{16} \mathrm{INO}_{4} \cdot 0.1 \mathrm{H}_{2} \mathrm{O}$ : C, 50.14; H, 3.41; N, 2.92. Found: C, 49.84; H, 3.11; N, 2.97.

\section{2-Iodo-3,4-dimethoxy- $N$-methyl- $N$-(6,7-methylenedioxy-1-naphthyl)benzamide (22)}

To a mixture of $\mathrm{NaH}(60 \%$ in mineral oil, $76 \mathrm{mg}, 1.89 \mathrm{mmol}$ ) and DMF (10 mL), 21 (300 mg, 0.629 mmol) was added and stirred at rt for $30 \mathrm{~min}$. MeI $(1.57 \mathrm{ml}, 0.30 \mathrm{mmol})$ was added to the mixture, then stirred at $70{ }^{\circ} \mathrm{C}$ overnight. The mixture was poured into water and extracted with AcOEt. The organic layer was washed with brine, dried over $\mathrm{MgSO}_{4}$, and concentrated to give a residue. Silica gel column chromatography with AcOEt/Hexane (1:4) gave 22 (235 mg, 76\%). Colorless prisms, mp 189.9-190.4 ${ }^{\circ} \mathrm{C}$ (AcOEt-hexane). IR (KBr) cm ${ }^{-1}: 1654$ (CO), 1463(C-N), 1246 (COC). ${ }^{1} \mathrm{H}-\mathrm{NMR}\left(500 \mathrm{MHz}, \mathrm{CDCl}_{3}\right.$ ) $\delta$ : $7.68\left(0.15 \mathrm{H}, \mathrm{d}, J=8.5 \mathrm{~Hz}, \mathrm{C}_{6}-\mathrm{H}\right), 7.47\left(1 \mathrm{H}, \mathrm{d}, J=7.7 \mathrm{~Hz}, \operatorname{naphC}_{6}-\mathrm{H}\right) 7.35\left(1 \mathrm{H}, \mathrm{d}, J=7.7 \mathrm{~Hz}\right.$, naphC $\left._{8}-\mathrm{H}\right)$, $7.29\left(1 \mathrm{H}, \mathrm{s}\right.$, naphC $\left._{1}-\mathrm{H}\right) 7.10(1 \mathrm{H}, \mathrm{t}, J=7.7 \mathrm{~Hz}$, naphC $7-\mathrm{H}), 7.19,7.08\left(0.15,0.8 \mathrm{H}, \mathrm{s}\right.$, naphC$\left._{4}-\mathrm{H}\right), 7.03$ $\left(0.15 \mathrm{H}, \mathrm{d}, J=8 \mathrm{~Hz}, \mathrm{C}_{5}-\mathrm{H}\right), 6.61\left(0.85 \mathrm{H}, \mathrm{d}, J=8.5 \mathrm{~Hz}, \mathrm{C}_{6}-\mathrm{H}\right), 6.36\left(0.85 \mathrm{H}, \mathrm{d}, J=8.5 \mathrm{~Hz}, \mathrm{C}_{5}-\mathrm{H}\right), 6.11$, $6.09\left(1.7 \mathrm{H}, \mathrm{d}, J=1.5 \mathrm{~Hz}, \mathrm{OCH}_{2} \mathrm{O}\right), 6.08,6.05\left(0.3 \mathrm{H}, \mathrm{d}, J=1.5 \mathrm{~Hz}, \mathrm{OCH}_{2} \mathrm{O}\right) 3.93,3.90\left(0.45 \mathrm{H}, \mathrm{s}, \mathrm{OCH}_{3}\right)$, 3.73, 3.64 (2.55H, s, $\left.\mathrm{OCH}_{3}\right), 3.51$ (2.55H, s, $\left.\mathrm{NCH}_{3}\right), 3.21\left(0.45 \mathrm{H}, \mathrm{s}, \mathrm{NCH}_{3}\right) .{ }^{13} \mathrm{C}-\mathrm{NMR}\left(125 \mathrm{MHz}, \mathrm{CDCl}_{3}\right)$ $\delta: 170.8,152.1,148.94,148.91,148.0,139.4,135.2,131.6,127.3,127.1,124.4,124.2,122.4,111.3$, 104.5, 101.5, 99.3, 93.3, 60.3, 55.7, 37.3. Anal. Calcd for $\mathrm{C}_{21} \mathrm{H}_{18} \mathrm{INO}_{5}$ : C, 51.34; H, 3.69; N, 2.85. Found: C, 51.58; H, 3.66; N, 2.92.

\section{9,10-Dimethoxy- $N$-methyl-2,3-methylenedioxybenzo[c]phenanthridine-6(5H)-one (23)}

A mixture of 22 (500 mg, $1.02 \mathrm{mmol}$ ), $\mathrm{PPh}_{3}$ (107.4 mg, $0.408 \mathrm{mmol}$ ), $\mathrm{Ag}_{2} \mathrm{CO}_{3}$ (563 mg, $2.04 \mathrm{mmol}$ ) $\mathrm{Pd}(\mathrm{OAc})_{2}$ (45.8 mg, $0.036 \mathrm{mmol}$ ), and DMF (5 mL) was heated under reflux for $1 \mathrm{~h}$. After the mixture was diluted with AcOEt, any undissolved materials were filtered off. The mother liquid was washed with brine, dried over $\mathrm{MgSO}_{4}$, and concentrated to give a residue which was subjected to silica gel column chromatography with AcOEt-hexane (1:3). The title compound 23 (337 mg, 91\%) was obtained, which was recrystallized from AcOEt for use as an analytical sample. Colorless needles, mp 261.6-262.4 ${ }^{\circ} \mathrm{C}$ (AcOEt). IR (KBr) cm ${ }^{-1}: 1644$ (CO), 1464 (CN), 1321, 1040 (COC). ${ }^{1} \mathrm{H}-\mathrm{NMR}\left(500 \mathrm{MHz}, \mathrm{CDCl}_{3}\right)$ $\delta: 9.10$ $\left(1 \mathrm{H}, \mathrm{d}, J=8.8 \mathrm{~Hz}, \mathrm{C}_{11}-\mathrm{H}\right), 8.38\left(1 \mathrm{H}, \mathrm{d}, J=8.8 \mathrm{~Hz}, \mathrm{C}_{7}-\mathrm{H}\right), 7.538\left(1 \mathrm{H}, \mathrm{d}, J=8.8 \mathrm{~Hz}, \mathrm{C}_{12}-\mathrm{H}\right), 7.535(1 \mathrm{H}, \mathrm{s}$, $\left.\mathrm{C}_{4}-\mathrm{H}\right), 7.20\left(1 \mathrm{H}, \mathrm{d}, J=8.8 \mathrm{~Hz}, \mathrm{C}_{8}-\mathrm{H}\right), 7.15\left(1 \mathrm{H}, \mathrm{s}, \mathrm{C}_{1}-\mathrm{H}\right), 6.08\left(2 \mathrm{H}, \mathrm{s}, \mathrm{OCH}_{2} \mathrm{O}\right), 4.02\left(3 \mathrm{H}, \mathrm{s}, \mathrm{NCH}_{3}\right), 3.89$ $\left(6 \mathrm{H}, \mathrm{s}, \mathrm{OCH}_{3}\right) .{ }^{13} \mathrm{C}-\mathrm{NMR}\left(125 \mathrm{MHz}, \mathrm{CDCl}_{3}\right) \delta: 164.7,156.8,147.9,146.7,145.6,136.9,132.1,128.0$, 125.8, 123.1, 122.8, 120.8, 120.1, 117.1, 112.2, 104.3, 102.8, 101.5, 60.3, 56.2, 41.7. HRMS (EI) m/z: calcd for 363.1107, found: 363.1109. 


\section{9,10-Dimethoxy- $N$-methyl-2,3-methylenedioxybenzo[c]phenanthridinium chloride (2)}

To a solution of 23 (100 mg, $0.275 \mathrm{mmol})$ in THF (5 ml), $\mathrm{LiAlH}_{4}(31.3 \mathrm{mg}, 0.825 \mathrm{mmol})$ was added at $0{ }^{\circ} \mathrm{C}$, then the mixture was vigorously stirred for $30 \mathrm{~min}$. After quenching with ice water, any undissolved materials were filtered off. To the mother liquid, a $10 \% \mathrm{HCl}$ aqueous solution was added to leave yellow precipitates which were recrystallized from $\mathrm{MeOH}$. The title compound 2 (78 mg, 74\%) was obtained as yellow needles, mp 195.6-196.1 ${ }^{\circ} \mathrm{C}(\mathrm{MeOH})$ [lit $\left.{ }^{8 \mathrm{~b}} 152-153{ }^{\circ} \mathrm{C}\left(\mathrm{CHCl}_{3}-\mathrm{MeOH}\right)\right]$. IR $(\mathrm{KBr}) \mathrm{cm}^{-1}: 1619$ $(\mathrm{C}=\mathrm{N}), 1495$ (CN), 1285, 1259 (COC). ${ }^{1} \mathrm{H}-\mathrm{NMR}\left(500 \mathrm{MHz}, \mathrm{CD}_{3} \mathrm{OD}\right) \delta: 9.77\left(1 \mathrm{H}, \mathrm{s}, \mathrm{C}_{6}-\mathrm{H}\right), 9.16(1 \mathrm{H}, \mathrm{d}$, $\left.J=9.3 \mathrm{~Hz}, \mathrm{C}_{11}-\mathrm{H}\right), 8.33\left(1 \mathrm{H}, \mathrm{d}, J=9.3 \mathrm{~Hz}, \mathrm{C}_{7}-\mathrm{H}\right), 8.00\left(1 \mathrm{H}, \mathrm{s}_{4} \mathrm{C}_{4}-\mathrm{H}\right), 7.95\left(1 \mathrm{H}, \mathrm{d}, J=9.3 \mathrm{~Hz}, \mathrm{C}_{12}-\mathrm{H}\right)$, $7.88\left(1 \mathrm{H}, \mathrm{d}, J=9.3 \mathrm{~Hz}, \mathrm{C}_{8}-\mathrm{H}\right), 7.36\left(1 \mathrm{H}, \mathrm{s}, \mathrm{C}_{1}-\mathrm{H}\right), 6.24\left(2 \mathrm{H}, \mathrm{s}, \mathrm{OCH}_{2} \mathrm{O}\right), 4.81\left(3 \mathrm{H}, \mathrm{s}, \mathrm{NCH}_{3}\right), 4.18$ (3H, s, $\mathrm{OCH}_{3}$ ), $3.93\left(3 \mathrm{H}, \mathrm{s}, \mathrm{OCH}_{3}\right) .{ }^{13} \mathrm{C}-\mathrm{NMR}\left(125 \mathrm{MHz}, \mathrm{CD}_{3} \mathrm{OD}\right) \delta: 162.2,155.7,151.0,150.1,145.9,134.4$, 134.1, 132.1, 130.9, 129.1, 125.4, 122.9, 121.2, 120.5, 118.1, 106.1, 105.0, 104.2, 60.9, 57.8, 52.1. Anal. Calcd for $\mathrm{C}_{21} \mathrm{H}_{18} \mathrm{ClNO}_{4} \cdot 0.8 \mathrm{H}_{2} \mathrm{O}$ : C, 63.34; H, 4.96; N, 3.52. Found: C, 63.38; H, 5.10; N, 3.59.

\section{ACKNOWLEDGEMENTS}

The authors thank the SC-NMR Laboratory of Okayama University for the NMR experiments. Part of this work was financially supported by JSPS KAKENHI (Grant No. 15 K07854 for H. A.).

\section{REFERENCES AND NOTES}

1. For reviews, see: (a) J. Hassan, M. Sévignon, C. Gozzi, E. Schulz, and M. Lemaire, Chem. Rev., 2002, 102, 1359; (b) D. Alberico, M. E. Scott, and M. Lautens, Chem. Rev., 2007, 107, 174; (c) M. Livendahl and A. M. Echavarren, Isr. J. Chem., 2010, 50, 630; (d) J. Yamaguchi, A. D. Yamaguchi, and K. Itami, Angew. Chem. Int. Ed., 2012, 51, 8960.

2. (a) T. Harayama, Heterocycles, 2005, 65, 697; (b) H. Abe and T. Harayama, Heterocycles, 2008, 75, 1305.

3. (a) S. D. Phillips and R. N. Castle, J. Heterocycl. Chem., 1981, 18, 223; (b) M. Suffiness and G. Cordell, The Alkaloids, Vol. 25, ed. by A. Brossi, Academic Press, New York, 1985, 178; (c) V. Simanek, The Alkaloids, Vol. 26, ed. by A. Brossi, Academic Press, New York, 1985, 185; (d) J. Dostal and M. Potacek, Collect. Czech. Chem. Commun., 1990, 55, 2840; (e) J. M. Herert, J. Augereau, J. P. Grleye, and J. Maffrand, Biochem. Biophys. Res. Commun., 1990, 172, 993; (f) S.-D. Fang, L.-K. Wang, and S. M. Hecht, J. Org. Chem., 1993, 58, 5025; (g) C. Vavreckova, I. Gawlik, and K. Müller, Planta Med., 1996, 62, 397; (h) S. P. MacKay, O. Meth-Cohn, and R. D. Waigh, Adv. Heterocycl. Chem., 1997, 67, 345; (i) T. Schmeller, B. Latz-Bruning, and M. Wink, Phytochemistry, 1997, 44, 257; (j) T. Nakanishi and M. Suzuki, J. Nat. Prod., 1998, 61, 1263; (k) T. Ishikawa and H. Ishii, Heterocycles, 1999, 50, 627; (l) T. Nakanishi, M. Suzuki, A. Saimoto, and T. 
Kabasawa, J. Nat. Prod., 1999, 62, 864, and references cited therein; (m) F. Fleury, A. Sukhanova, A. Ianoul, J. Devy, I. Kudelina, O. Duval, A. Alix, J. Jardillier, and I. Nabiev, J. Biol. Chem., 2000, 275, 3501; (n) L. C. Tavares, G. Zanon, A. D. Weber, A. T. Neto, C. P. Mostardeiro, I. B. M. Da Cruz, R. M. Oliveira, V. Ilha, I. I. Dalcol, and A. F. Morel, Plos One, 2014, 9, e97000.

4. (a) T. Harayama, H. Akamatsu, K. Okamura, T. Miyagoe, T. Akiyama, H. Abe, and Y. Takeuchi, J. Chem. Soc., Perkin Trans. 1, 2001, 523; (b) T. Harayama, T. Akiyama, H. Akamatsu, K. Kawano, H. Abe, and Y. Takeuchi, Synthesis, 2001, 444; (c) T. Harayama, T. Akiyama, Y. Nakano, K. Shibaike, H. Akamatsu, A. Hori, H. Abe, and Y. Takeuchi, Synthesis, 2002, 237; (d) T. Harayama, T. Sato, Y. Nakano, H. Abe, and Y. Takeuchi, Heterocycles, 2003, 59, 293; (e) T. Harayama, T. Sato, A. Hori, H. Abe, and Y. Takeuchi, Synthesis, 2004, 1446.

5. N. F. DeMoura, H. B. Ribeiro, E. C. S. Machado, E. M. Ethur, N. Zanatta, and A. D. Morel, Phytochemistry, 1997, 46, 1443.

6. S.-Q. Pang, G.-Q. Wang, B.-K. Huang, Q.-Y. Zhang, and L.-P. Qin, Chem. Nat. Compds., 2007, 43, 100 .

7. $\quad$ K. W. Bentley, Nat. Prod. Rep., 1999, 16, 367.

8. (a) H. Abe, N. Kobayashi, Y. Takeuchi, and T. Harayama, Heterocycles, 2010, 80, 873; (b) Y. Ishihara, S. Azuma, T. Choshi, K. Kohno, K. Ono, H. Tsutsumi, T. Ishizu, and S. Hibino, Tetrahedron, 2011, 67, 1320.

9. For a preliminary communication of a part of this work, see: ref. 8a.

10. J. Smidrkal, Coll. Czech. Chem. Commun., 1988, 53, 3184.

11. F. R. Stermitz, J. P. Gillespie, L. G. Amoros, R. Romero, and T. A. Stermitz, J. Med. Chem., 1975, 18, 708 .

12. (a) S. Manna, J. R. Falck, and C. Mioskowski, J. Org. Chem., 1982, 47, 5021; (b) S. V. Kessar, Y. P. Gupta, P. Balakrishnan, K. K. Sawal, T. Mohammad, and M. Dutt, J. Org. Chem., 1988, 53, 1708.

13. (a) H. Harayama, H. Toko, K. Kawata, H. Nishioka, H. Abe, and Y. Takeuchi, Heterocycles, 2002, 58, 175; (b) T. Matsukihira, T. Kida, K. Hidaka, S. Saga, M. Takemura, A. Yonoki, T. Nishimori, Y. Horino, T. Harayama, Y. Takeuchi, and H. Abe, Heterocycles, 2013, 87, 2555; (c) T. Matsukihira, S. Saga, Y. Horino, and H. Abe, Heterocycles, 2014, 89, 59.

14. K. M. Markovich, V. Tantishaiyakul, A. Hamada, D. D. Miller, K. J. Romstedt, G. Shams, Y. Shin, P. F. Fraundorfer, K. Doyle, and D. R. Feller, J. Med. Chem., 1992, 35, 466.

15. (a) L. C. Raiford and E. H. Wells, J. Am. Chem. Soc., 1935, 57, 2500; (b) S. B. Jones, L. He, and S. L. Castle, Org. Lett., 2006, 8, 3757.

16. N. T. T. Chau, T. H. Nguyen, A.-S. Castanet, K. P. P. Nguyen, and J. Mortier, Tetrahedron, 2008, 64, 10552. 